\title{
From habitat use to social behavior: natural history of a voiceless poison frog, Dendrobates tinctorius
}

Rojas, B. \& Pašukonis, A. 2019. PeerJ.

\section{Treefall attendance}

BR witnessed the formation of nine tree-fall gaps over the study periods (one in 2009, eight in 2011); these were discovered rapidly because they occurred in the $1.5 \mathrm{~km}$ transect surveyed daily. BR inspected each gap within the first 24 hours of its formation and caught as many frogs as possible, moving fallen branches until no frogs were seen (after 2-3 hours). During the next two consecutive days BR carefully searched for new frogs over a similar period of time $(2-3 \mathrm{~h})$. When frogs were seen but not caught, BR photographed them from a distance to record their color pattern for further identification upon capture. Two days after treefall occurrence, one, two or three bowls with water were added at six of the newly formed gaps (depending on the gap's size) during the course of a parallel study (Rojas 2015). These bowls were meant to simulate newly available tadpole-deposition sites.

Data file name: Rojas\&Pašukonis2019PeerJ_DataOnTrefallAttendance Variable description:

frogid = individual id for each frog, based on the uniqueness of their colour patterns sex $=$ the sex of each frog latency_TF = time taken for each individual frog to arrive in a new treefall gap

Each row represents one frog individual.

\section{Tadpole transport}

Males carrying tadpoles were found during daily surveys along a $1.5 \mathrm{~km}$ transect. BR recorded the number of tadpoles on the back of each tadpole-carrying male and captured it when possible. Upon capture, each male was photographed (with the tadpole(s) still attached) against graph paper. Later these photos were used to measure the size of both the frog and the tadpoles with the software ImageJ (Schneider et al. 2012). Tadpole size was measured dorsally, from the tip of the snout to the base of the tail. 
Data file name: Rojas\&Pašukonis2019PeerJ_DataOnTadpoleTransport

Variable description:

male_id = individual id of each male found with tadpoles on its back

numbertads $=$ number of tadpoles transported by a single male

tadpole_id $=$ tadpole identity

tadsize $=$ tadpole size in $\mathrm{mm}$ (see methods of measurement above)

Each row represents one tadpole.

\section{Habitat use}

During the field season of 2010, BR captured 109 frogs (55 females and 54 males), each of which was assigned to one of two microhabitats according to where they were first seen: leaf litter (when frogs were on a relatively open patch of leaf litter without any obvious structure in a 1 m radius), or associated to the following structures: fallen logs (when frogs were visibly exposed on top of the log or inside hollow trunks), fallen branches (when individuals were in fallen tree crowns) and tree roots (when the frogs were within the exposed roots or next to them). Frogs were only included in the analyses once (recaptures of the same individual were excluded in order to avoid pseudoreplication, and only the site at first sighting was taken into account). We tested for differences between the sexes in the microhabitat where they were found (open vs. associated with the aforementioned structures) using a Generalized Linear Model with binomial distribution. All statistical analyses were done with the software R v. 3.3.3 (RCoreTeam 2013) using the RStudio interface (RStudio 2015).

Data file name: Rojas\&Pašukonis2019PeerJ_DataOnHabitatUse

Variable description:

frogid = individual id for each frog, based on the uniqueness of their colour patterns sex $=$ the sex of each frog 
struct-habitat $=$ characteristic of the microhabitat in which every frog was found; either 0 (open leaf litter) or 1 (associated to structures such as those described in the methods above)

Each row represents one frog.

\section{Size at metamorphosis}

Metamorphs were found thanks to regular monitoring of pools. Size at metamorphosis was measured for 24 individuals using the software ImageJ.

Data file name: Rojas\&Pašukonis2019PeerJ_DataOnSizeAtMetamorphosis

Variable description:

froglet_id = individual id for each frog in association to the id of the pool where they emerged from

size $=$ metamorph size in $\mathrm{mm}$ (see methods of measurement above)

Each row represents one metamoprh. 